REVIEW

\title{
CURRENT MORPHOLOGICAL AND IMMUNOHISTOCHEMICAL FEATURES OF MALE BREAST CANCER
}

\author{
A. C. Tutovanu ${ }^{1}$, G. Drăghici ${ }^{2}$, S. M. Oprescu ${ }^{1,3}$ \\ ${ }^{1}$ Carol Davila University of Medicine and Pharmacy, Bucharest, Romania \\ 2Clinical Nephrology Hospital "Dr. Carol Davila", Bucharest, Romania \\ 3University Emergency Hospital of Bucharest, Romania \\ Corresponding author: Tutovanu Andrei Costin \\ Phone no.: +40744162946 \\ E-mail: tutovan@drd.umfcd.ro
}

\begin{abstract}
Breast cancer continues to be a leading cause of death among women aged over 40 years. This type of neoplasm is diagnosed quite rarely in male sex over 60 years, with an incidence almost 100 times lower in men versus women. No definite cause of occurrence of this ailment has been yet discovered in male sex, as well as in the case of breast cancer in female sex, but both environmental and genetic factors are incriminated. It appears that BRCA2 mutations are associated with increased risk of male breast cancer $(M B C)$ and invasive ductal carcinoma is the predominant histological type of disease, which is positivity of both estrogen and progesterone receptors. This review was made with an aim to analyze morphological and immunohistochemical features on male breast cancer. PubMed and Medscape were used to identify systematic reviews published using the title term "molecular subtype of male breast cancer".
\end{abstract}

Keywords: male breast cancer, invasive ductal carcinoma, ER receptor

\section{Introduction}

Breast cancer in men (MBC) is a rare malignant disease, only about $1 \%$ of all cases being diagnosed worldwide [1]. There are biological differences between male and female sex, that are mentioned in the literature (PubMed), but they are not related to morphology in such an important measure. The morphological similarity of the two sexes from the neoplastic point of view is mainly represented by the ductal carcinoma of the infiltrative type. MBC is diagnosed at a much older age, at an advanced TNM stage associated with the enlargement and greater involvement of lymph nodes versus FBC [2].
Since the MBC occurs quite rarely, treatment strategies are based in particular on the therapeutic methods used in the case of female breast cancer (FBC). With the development of targeted agents against human epidermal growth receptor (HER-2) such as: lapatinib, trastuzumab, pertuzumab or hormonal agents (aromatase inhibitors), the results of treatment in FBC had a substantial improvement. In the absence of clinical trials, these drug-type treatments could not be fully implemented in the case of MBC [3].

Despite the treatment, between 30 and 50\% of male patients known to have breast cancer at an advanced TNM stage, will suffer at least one bone metastasis with a significant decrease in quality of life. Only $20 \%$ of patients who have 
already been diagnosed with at least one bone metastasis, will survive more than 5 years. Breast cancer cells once reached at the level of the bone microenvironment, will activate the osteoclasts or/and osteoblasts that will lead to bone destruction or bone apposition [4].

\section{Materials and Methods}

This paper aims to make a review of the notions in the literature about the current morphological and immunohistochemical features of male breast cancer and is based on the analysis of the results from the databases: Web of Science, Scopus and PubMed. The search used the formula: "male breast cancer" AND ("morphology" OR "immunohistochemistry") and have been filtered according to the language (English) and publication year.

\section{Results}

\section{BRCA1 and BRCA2 genes}

The MBC is different from the FBC, due to the mutations occurring in the BRCA1 and BRCA2 genes. Mutations of the BRCA1 gene occur in $0-4 \%$ of cases, and those in the BRCA2 gene are more commonly encountered in males, in a percentage of $4-16 \%$ of cases. Moreover, these mutations appear at a much lower age with a poorly and reserved prognosis [5].

BRCA1 and BRCA2 are tumor suppressor genes that play a role in encoding DNA repair proteins. More than 1,200 mutations in the BRCA1 gene, respectively 1,300 mutations of the BRCA2 gene, were reported in the literature. Patients that have a defective allele BRCA1 or BRCA2 and in addition inherit the second fault allele from parents (mother or father) would develop a neoplasm, through an accumulation of additional mutations [6]. Mutations of the BRCA1 gene (located at the level of chromosome 17q21) are represented by the occurrence of STOP codons at the reading frame level, the result being a truncated type protein. The most common mutations are 185 delAG (leading to the formation of the STOP codon in the amino acid 39, in the protein sequence), respectively 188del11, which modifies the reading framework in the exon level 3, with the formation of a truncated protein. The repetitive type ALU sequences are characteristic elements for the gene BRCA1 [7]. The rearrangement is uncommon in the case of BRCA2 gene (located in the 13q12-q13 chromosome). From the 229th nucleotides level, the reading framework would begin, coding a protein of $390 \mathrm{kDa}$. BRCA2 has a functioning role similar to that of a repairing protein, from the DNA level, by interacting with the RAD51 tumor suppressor. Most mutations consist of defects in the reading framework and in the occurrence of non-sense mutations at the level of exon 11, 617delT, being the most common mutation encountered [8].

Both BRCA genes are made up of complex genomic structures. BRCA1 is composed of 24 exons, and BRCA2 of 27 exons. They both have a role in protein coding, BRCA1 codifies a protein consisting of 1863 amino acid and BRCA2 a protein consisting of 3418 amino acids [9].

However, there is quite little information on the pathology of breast cancer in men with mutations in genes BRCA1/2. Breast cancer in men is rarely representing less than $1 \%$ of all male neoplasms [10].

\section{Pathology and Immunohistochemistry}

Approximately $90 \%$ of breast cancer cases occurring in male sex are of the type of ductal invasive carcinoma. The ductal invasive carcinoma is a solid, whitish grey tumor, imprecisely delimited, with extensions in the adjacent adipose tissue. It has a high, hard consistency due to calcification, respectively the desmoplastic reaction. Rarely, the tumor may present soft consistency and well delimited edges. Dimensions range from 2 to $5 \mathrm{~cm}$ [11].

The remaining carcinoma of the male mammary gland (less than 1,5\%) are originated from the lobular epithelium. Invasive lobular carcinoma is characterized by the presence of cells arrangement, in a row, occasionally having a vacuole filled with perinuclear mucus, they may also present intense fibrosis reactions (desmoplastic reactions) [12].

In most cases of breast cancer, high levels of hormonal receptors may be observed. Estrogen and progesterone receptors are more likely to be positive for male than for females. The 
proportion of hormone receptor tumors can increase with aging [13].

Approximately $90 \%$ of breast cancer cases occurring in male sex have estrogenic receptors, respectively $81 \%$ progesterone. However, the overexpression of the HER2/neu oncogene, a useful prognostic marker, of breast cancer in female sex, is not always found in male cases. The role of the androgen receptor in male breast cancer is unclear, as the literature specifies, the expression of this receptor varies between 34 and $95 \%$, without influencing the prognosis [14].

It is known that $\mathrm{MBC}$, like the $\mathrm{FBC}$, has positive androgenic receptors, hence these receptors of steroidal hormones may be considered an interesting target for medicines that may also block estrogen receptors. Androgen receptor therapy, according to the latest PubMed and specialty literature studies, is tested on both male and female subjects, both in monotherapeutical and as a combination of therapy with alpha estrogenic receptor inhibitors. The biological interaction between androgenic and estrogenic receptors in the case of MBC is still completely unexplored [15].

Immunohistochemistry is used in the classification of breast cancer, because routine use of the analysis of the microarray gene expression is not possible [16].

Estrogenic and progesteronic receptors are found at the level of the breast epithelium, both normal and neoplastic type. Estrogens play a role in the synthesis of progesterone, therefore, tumors of type RE + (estrogen receptor positive) may occur, respectively RP- (negative progesterone receptor) [17].

Oncogene HER-2 / neu (from chromosome 17) is responsible of encoding the synthesis of a transmembraneous protein with a molecular weight of $185 \mathrm{kDa}$, this being part of the tyrosine kinase receptor family. The same structural and functional correspondence has the epidermal growth factor receptor (EGFR) with tyrosine kinase receptors. HER1 and HER2 are associated with the development of breast cancer. The HER2 receptor is linked to growth factors, which produce both growth and cell division. The overexpression of HER-2/neu oncogene is encountered in breast cancer, generating a continuous cellular division [18].
EGFR is a signaling molecule, involved in both biological and neoplastic processes. It is one of the most studied signaling pathways involved in the development of breast cancer. EGFR expression, in breast tumors metastases is high, correlated with an aggressive phenotype and a poor survival prognosis. The epidermal growth factor receptor (EGFR) was first discovered in the ErbB family of tyrozin-kinazic receptors, which includes a total of four members: ErbB1/He1, ErbB2/Her-2/neu, ErbB3/Her3 and ErbB4/Her4. In breast cancer, activation of aberrant EGFR contributes to the transformation of normal cells into neoplastic cells, respectively, to the invasion and progression of the primary breast tumors [19].

The P53 gene is a recessive gene, located at the level of chromosome 17p13. By losing both alleles, the expression of the P53 gene will be lost. In breast cancer, the mutation of the P53 gene is associated with a more aggressive disease with a low survival rate [20]. The mutation of the P53 gene results in the passage of breast neoplasia from preinvasive forms to invasive type. Lack of gene P53 causes loss of cell cycle control, of DNA replication, the fidelity of DNA replication and cellular apoptosis, so the cells with DNA lesions will survive and the tumor progression will occur [21].

The Ki-67 antigen is a marker of cellular cycle and cellular proliferation, used to estimate the proliferation coefficient in a cellular population. The Ki-67 antigen is a nuclear antigen expressed in all phases of the cellular cycle, but not in phase G0. In breast neoplasia, the percentage of positive $\mathrm{Ki}-67$ cells is correlated with the parameters of aggressiveness, of tumor progression, of resistance to endocrine or chemotherapeutic treatment [22].

Citokeratins are supportive proteins, that form the cellular cytoskeleton together with microtubules and actin filaments. Citokeratins contain 20 different polypeptides and are histopathological recognized, as intermediate filaments with a role in differentiating healthy tissues from the pathological ones. CK18 together with $\mathrm{CK} 8$ are proteins that are expressed in adult epithelial organs (breast), and can also be expressed through tumors developed from the level of these breast tissues [23]. 
Cytokeratins have some distinct advantages over marker proteins. Some researchers have shown that, primary mammary neoplasms with metastatic metastases, have the same cytokeratin, such as normal breast epithelium. The tumor expression of CK8 and CK18 were observed in over $90 \%$ of cases of breast carcinoma, thus the efficacy of immunohistochemical identification of breast cancer can be confirmed [24].

The most differentiated breast cancers are of type RE +, HER2-and RP +, while poorly differentiated tumors are HER2 +, RE-and RP-.

Studies using the genetic expression profile identified five major molecular subtypes of breast cancer: "luminal A", "luminal B", "HER2 group", "normal breast-like" group and "basal cell carcinoma" group.

The other molecular subtypes, such as "luminal C", respectively "normal breast like" have been identified in some specialized studies [25].

- Category "luminal A" includes RE (+), RP (+) and HER2 (-) and Ki-67 <14\% tumors. The luminal (+) subtype is better described and characterized by a panel of immunohistochemical markers: RP, RE, CK8.

- Category "luminal B" includes tumors of type RE (+), RP (+) and HER2 (+), RE $(+)$, RP (+), HER2 (-) and Ki-67 > 14\%.

- The "HER2" group includes RE (-), RP $(-)$ and HER2 (+) tumors.

- Triple negative group includes RE (-), RP (-) and HER2 (-) tumors.

- Basal group includes RE (-), RP (-), HER2 (-) and basal marker (+) tumors.

- Normal like includes RE (+), RP (+), HER2 (-), Ki67 (-) tumors.

At the present, the clinical significance of the genetic expression or the immunophenotypical profile in the case of other types of breast cancer time, it is not known. Invasive ductal carcinoma is an exception [26].

\section{Breast cancer in male vs. female sex}

The majority of breast cancers occurring in male sex are ER positive, in contrast to the female sex, the expression of the ER receiver is a marker of differentiation, indicating that breast cancer is still under hormone influence, with low aggression and maximum responsiveness to hormonal type therapy [27].

In the literature there are few studies, focusing on the fact that, the level of estrogen is low in the body, but increased intratumorally in men. This fact is the question of whether men will have the same survival rate, if they benefit from the same endocrine treatment prescribed to postmenopausal women with tumor cancer (low estrogen level, with increased ER receptor expression) [28].

HER2 expression in breast cancer, in the male sex is controversial according to the specialty literature, men with HER2-positive breast cancer have a reserved prognosis towards women. Therapies for breast cancer in male and female sex have varied over the last decade by altering survival rates for both sexes [29].

\section{Conclusion}

MBC in contrast to FBC is rare and little research has been done. There is a substantial need to obtain more information about the anatomopathology and immunohistochemistry of $\mathrm{MBC}$, as well as modern methods of treatment. The predominant histological type in MBC is invasive ductal carcinoma. BRCA1 and more frequently, BRCA2 mutations are associated with MBC. The high levels of hormonal receptors (ER, PR) and the overexpression of the HER2/neu oncogene, makes it attractive for endocrine and Her2 therapies. Most research data on MBC are drawn from retrospective studies, thus are essential more studies from different oncology centers for a better understanding of optimal treatment approaches and to determine a specific treatment protocol. Education of male population is needed to increase knowledge of breast cancer and to encourage registration onto future studies, in case of MBC.

\section{References}

[1]RM. Ferzoco, Kh. J. Ruddy, "Optimal Delivery Of Male Breast Cancer Follow-Up Care: Improving 
Outcomes Breast Cancer," Dove Med Pres. Published Online, pp. 371-379, Nov 2015.

[2]G Masci, $M$ Caruso, $F$ Losurdo, et al "Clinicopathological And Immunohistochemical Characteristics In Male Breast Cancer: A Retrospective Case Series," Oncologist. Published Online, pp. 586-592, Jun 2015.

[3]YH Hong, K S Ha, YH Jung, et al "Clinical Features Of Male Breast Cancer: Experiences From Seven Institutions Over 20," Cancer Res Treat. Published Online, pp.1389-1392, Apr 2016.

[4]S Shemanko, Y Cong, AFA Desiderio "What Is Breast In The Bone?," Academic Editorint J Mol Sci. , pp.1758-1764, Oct 2016.

[5]A Chau, N Jafarian, M Rosa "Male Breast: Clinical and Imaging Evaluations of Benign and Malignant Entities with Histologic Correlation.," Am J Med. Published online, pp. 776-791, Aug 2016.

[6]P Prolla, I Ewald, I Riberp, et al " Genomic Rearrangements In Brca1 And Brca 2: A Literature Review," In Genetics And Molecular Biology, pp.437-446, Mar 2009.

[7]N Mavaddat, D Barrowdale, IL Andrulis, et al "Pathology of breast and ovarian cancers among BRCA1 and BRCA2 mutation carriers: results from the Consortium of Investigators of Modifiers of BRCA1/2 (CIMBA)," Cancer Epidemiol Biomarkers Prev. pp. 134-147, Sept 2012.

[8]G. Chevenix-Trench, RL Milne, AC Antoniou, et al "An international initiative to identify genetic modifiers of cancer risk in BRCA1 and BRCA2 mutation carriers: the Consortium of Investigators of Modifiers of BRCA1 and BRCA2 (CIMBA)," Breast Cancer Res. pp. 91-104, Oct 2007.

[9]E Van Der, PJ Van "Pathology Of Hereditary Breast Cancer," Cell Oncol (Dordr), pp. 71-88, Feb 2011.

[10]DG Evans, I Susnerwala, J Dawson, et al "Risk of breast cancer in male BRCA2 carriers," . J Med Genet, pp.710-711, Jan 2010.

[11]C Rudlowski "Male Breast Cancer," Breast Care (Basel). pp.183-189,Dec 2008.

[12]M Koc, S Oztas, MT Erem, et al. "Invasive lobular carcinoma of the male breast: a case report," Jpn J Clin Oncol. pp.444-446, Apr 2001.

[13]B Comet, B Cutuli, F Penault-Llorca "Male breast cancer: a review," Bull. Cancer. pp.181-189, May 2009.

[14]SH Giordano "A review of the diagnosis and management of male breast cancer,"Oncologist. pp.471-479, Jul 2005.

[15]Te M Sever-Son, W Zwart "A Review of Estrogen Receptor/Androgen Receptor Genomics in male breast cancer," Society For Endocrinology. pp.6-15, Apr 2017.
[16]Li Huiling,, Y Yng,, D Wang, et al "Molecular subtypes of male breast cancer by immunohistochemistry," Int J Clin Exp Med. pp. 1670-1677, Aug 2017.

[17]TM Severon, E Nevedomskaya, J Peeters, et al "Neoadjuvant tamoxifen synchronizes ER $\alpha$ binding and gene expression profiles related to outcome and proliferation," Oncotarget 7. pp. 33901-33918, Nov 2016.

[18]A Nashed, M Shaima, M Yussif "Ki-67 As A Prognostic Marker According To Breast Cancer Molecular Subtype," Cancer Biolmed.. pp. 496-504, Dec 2016.

[19]A Remah, MK Wendt "The Paradoxical Functions Of Egfr During Breast Cancer Progression," Signal Transduct Target Ther, pp.16042-16044, Jan 2017.

[20]D Walerych, M Napoli, L Collavin "The rebel angel: mutant p53 as the driving oncogene in breast cancer," Carcinogenesis, pp.2007-2017, Nov 2012.

[21]T Li., et al. "Tumor Suppression in the Absence of p53-Mediated Cell-Cycle Arrest, Apoptosis and Senescence," Cell, pp. 1269-1283, Feb 2012.

[22]A Nashed, M Shaima, M Yussif "Ki-67 As A Prognostic Marker According To Breast Cancer Molecular Subtype,"Cancer Biolmed.. pp. 496-504, Dec 2016.

[23]A Havam, M Samaka, Y Nancy, et al "Relationship Of Ck8/18 Expression Pattern To Breast Cancer Immunohistochemical Subtyping In Egyptian Patients," Ecancermedicalscience, pp. 404412, Aug 2014.

[24]MM Shao, SK Chan, AM Yu, et al "Keratin Expression In Breast Cancers," Virchows Arch, pp.313-322, Sept 2012.

[25]A Goldhirsch, WC Wood, AS Coates, et al "Strategies for subtypes-dealing with the diversity of breast cancer: highlights of the St. Gallen International Expert Consensus on the Primary Therapy of Early Breast Cancer," Ann Oncol. pp. 1736-1747, Oct 2011.

[26]M Smid, Y Zhang, AM Sieuwert, et al. "Subtypes of breast cancer show preferential site of relapse," Cancer Res. pp.3108-3114, Sept 2008.

[27]D Muir, R Kanthan, SC Kathan "Male versus Female breast cancers a population-based comparative immunohistochemical analysis," Archives of Pathology \& Laboratory Medicine, pp.36-41 Jan 2003.

[28]TM Severson, W Zwart "A review of estrogen receptor/androgen receptor genomics in male breast cancer." In endocrin related cancer, pp.r27-r34, Mar 2017.

[29]D Ly, D Forman, J Ferlay, et al “. An international comparison of male and female breast cancer incidence rates," Int J Cancer, pp.1918-1926, Aug 2013. 
